\title{
1 Effects of perfluoroalkyl and polyfluoroalkyl substances on soil
}

\section{2 structure and function}

3 Baile $X u^{1,2}$ *, Gaowen Yang ${ }^{1,2}$, Anika Lehmann ${ }^{1,2}$, Sebastian Riedel ${ }^{3}$, Matthias C. Rillig ${ }^{1,2}$

$4{ }^{1}$ Institute of Biology, Freie Universität Berlin, D-14195 Berlin, Germany

$5 \quad{ }^{2}$ Berlin-Brandenburg Institute of Advanced Biodiversity Research, D-14195 Berlin, Germany

$6{ }^{3}$ Institute of Chemistry and Biochemistry, Freie Universität Berlin, D-14195 Berlin, Germany

7

$8 \quad{ }^{*}$ Corresponding author. E-mail: bxu@zedat.fu-berlin.de 


\section{Abstract Art}

\section{Soil microcosm}

10

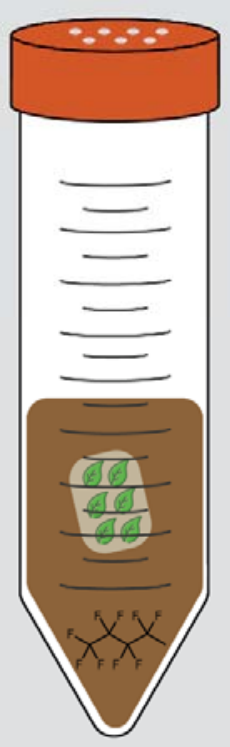

Per- and polyfiluoroalkyl substances

\section{Litter} decomposition

Positive
effect

Baterial abundance

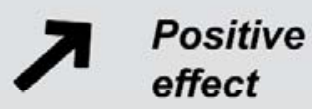

$\mathrm{BO}_{2} \quad$ Soil

$\triangle$ respiration

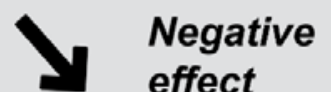

effect

Water-stable aggregates

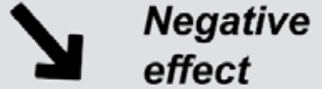




\section{Abstract}

13 Soils are impacted at a global scale by several anthropogenic factors, including chemical

14 pollutants. Among those, perfluoroalkyl and polyfluoroalkyl substances (PFAS) are of

15 concern due to their high environmental persistence, and as they might affect soil health and

16 functions. However, data on impacts of PFASs on soil structure and microbially-driven

17 processes are currently lacking. This study explored the effects of perfluorooctanesulfonic

18 acid (PFOS), perfluorooctanoic acid (PFOA) and perfluorobutanesulfonic acid (PFBS) at

19 environmental-relevant nominal concentrations $\left(1 \sim 1000 \mathrm{ng} \mathrm{g}^{-1}\right)$ on soil functions, using a 6-

20 week microcosm experiment. We measured soil respiration, litter decomposition, enzyme

21 and microbial activities, soil aggregates, and bacterial abundance. PFAS (even at $1 \mathrm{ng} \mathrm{g}^{-1}$

22 for PFBS) significantly increased litter decomposition, associated with positive effects on

23 bacterial abundance, and $\beta$-glucosidase activities. This effect increased with PFAS

24 concentrations. Soil respiration was significantly inhibited by PFAS in the $3^{\text {rd }}$ week, while this

25 effect was more variable in week 6 . Water-stable aggregates were negatively affected by

26 PFOS and PFOA, possibly related to microbial shifts. The general microbial activities and $\beta$ -

27 D-cellobiosidase and phosphatase activities were barely affected by PFAS treatments. Our

28 work highlights the potential effects of PFAS on soil health, and we argue that this substance

29 class could be a factor of environmental change of potentially broad relevance in terrestrial

30 ecosystem functioning.

31 Keywords: Litter decomposition; Soil respiration; Water-stable aggregates; Soil bacteria;

32 Perfluorobutanesulfonate (PFBS); Perfluorooctanesulfonic acid (PFOS); Perfluorooctanoic

33 acid (PFOA); Enzyme activities

35 Synopsis: PFAS are likely to affect soil health. 


\section{Introduction}

37 Human activity is progressively and fundamentally affecting the Earth's surface, including

38 soils, which operate at the interface between biosphere, hydrosphere, atmosphere, and

39 lithosphere, and are suffering from physical, chemical, and biological stressors related to

40 anthropogenic activities ${ }^{1,2}$. One significant category of these influences is chemical pollution,

41 which is widely recognized as a global change factor ${ }^{1,3}$. Perfluoroalkyl and polyfluoroalkyl

42 substances (PFAS) are a highly diverse family of chemicals of concern ${ }^{4}$. These compounds

43 contain the perfluoroalkyl moiety $\left(\mathrm{C}_{n} \mathrm{~F}_{2 n+1}\right)^{5}$, and the relatively high-energy carbon-fluorine

44 (C-F) bonds make them extremely resistant to breakdown, and subsequently persistent in

45 the environment. Therefore, they are also called "forever chemicals" in public discourse ${ }^{6}$.

46 Certain PFAS, perfluorooctanesulfonic acid (PFOS) and perfluorooctanoic acid (PFOA) are

47 on the list and on the waiting list under the Stockholm Convention ${ }^{7}$, respectively.

48 Soil is a major sink for persistent organic chemicals in the environment. There are many

49 pathways for PFAS entering the soil environment. Typically, fluoride factory emission, sludge

50 application, the degradation of aqueous film-forming foam, and landfills contribute direct

51 sources, and atmospheric deposition and runoff constitute non-point sources ${ }^{8,9}$. PFAS have

52 been widely detected in soils with a broad range of concentrations. Generally, PFAS

53 concentrations in non-hotspot soil are lower than $300 \mathrm{ng} \mathrm{g}^{-1}$, while in hotspots of PFAS-

54 contaminated soil, they can be as high as several or even tens of $\mu \mathrm{g} \mathrm{g}^{-1}$, mostly dominated

55 by PFOA and PFOS ${ }^{10}$. Jin et al. ${ }^{11}$ also reported that perfluorobutanesulfonic acid (PFBS)

56 was a prominent type of PFAS in the soil around a fluoride-factory park.

57 Existing evidence has demonstrated that PFAS can exert some impacts on soil functions, in

58 particular on soil enzymes, and microbial activities and communities, and these influences

59 were closely related to properties of soil and PFASs. According to the number of carbon

60 atoms, perfluoroalkyl carboxylic acids with 7 or more and perfluoroalkyl sulfonate with 6 or

61 more carbons are categorized as long-chain PFAS, otherwise, short-chain PFAS ${ }^{5}$. Short-

62 chain PFBS might activate sucrase and urease activities, while long-chain PFOS might 
63 reduce these activities in soil ${ }^{12}$. Cai et al. ${ }^{13}$ showed that PFAS with sulfonic groups and

64 longer chains had higher toxicity to soil microbial activities, and that soil with higher organic

65 matter content and higher $\mathrm{pH}$ (neutral) exhibited lower impact by PFAS. Differences in

66 sorption affinity of PFAS to soil are likely to regulate the impact of PFAS on soil microbes ${ }^{8}$.

67 Changes in structure and function of microbial communities by PFAS were shown by

68 previous research ${ }^{12,14-16}$, and these shifts were suggested to affect soil processes and

69 ecosystem functions. However, the implications on process rates in soil, for example, litter

70 decomposition and soil aggregation, were not addressed.

71 Given the persistent nature of PFAS it is important to explore if these chemicals can affect soil process rates and properties. Here, we investigate PFAS effects on soil processes,

73 including soil respiration, litter decomposition, soil aggregation, enzyme and microbial

74 activities, as well as bacterial abundance. We discuss the environmental implications of our

75 results and suggest that PFAS be considered as a global change factor of importance in

76 terrestrial ecosystems.

\section{Materials and Methods}

\section{Test soil and PFAS}

79 The test soil was Albic Luvisol collected at the agricultural field station of Freie Universität

80 Berlin in December 2020. The soil has a sandy loam texture $(73.6 \%$ sand, $18.8 \%$ silty and

$817.6 \%$ clay), with $1.87 \%$ total C, $0.12 \%$ total $\mathrm{N}$ and a soil $\mathrm{pH}$ of $7.1^{17,18}$. Fresh soil samples

82 were thoroughly mixed, passed through a 2-mm sieve, and then stored at $4^{\circ} \mathrm{C}$.

83 Three PFAS, namely perfluorooctanesulfonic acid (PFOS), perfluorooctanoic acid (PFOA)

84 and perfluorobutanesulfonic acid (PFBS) were selected in this study due to their wide

85 occurrence in the soil environment ${ }^{19}$. Values of their Log $K_{\text {ow }}$ are 5.26, 4.59 and 2.73,

86 respectively ${ }^{20}$, and their chemical structures and other physicochemical properties are listed

87 in Supporting Information (SI) Table S1. 
89 PFAS standards were dissolved in sterilized deionized water to prepare the stock solution

90 with the concentration of $100 \mathrm{mg} \mathrm{L}^{-1}$. A five-gram portion of previously sterilized soil samples

$91 \quad\left(121^{\circ} \mathrm{C}, 20 \mathrm{~min}\right.$, twice) was supplemented with appropriate doses of PFAS in solution (we

92 used this sterilized 'loading soil' to avoid any exaggerated effects on soil communities ${ }^{21}$ ),

93 and thoroughly mixed this soil with $25 \mathrm{~g}$ of soil by manual stirring for 2 mins. A total of $30 \mathrm{~g}$

94 soil was placed in 50-mL mini-bioreactor tubes (Corning Inc., Corning, USA) with vented lids

95 to establish experimental microcosms. Each tube was watered to $70 \%$ soil water holding

96 capacity $(\mathrm{WHC})$ with deionized water. The nominal concentrations of PFOS and PFOA in

97 soil were $1,10,100$ and $1000 \mathrm{ng} \mathrm{g}^{-1}$, and that of PFBS were $0.5,5,50$ and $500 \mathrm{ng} \mathrm{g}^{-1}$,

98 corresponding to their environmentally-relevant levels ${ }^{10}$. The analytical method and actual

99 concentrations of PFAS in soil are reported in SI Text S1, and Table S2, respectively. For

100 the convenience of comparison of three PFAS, we also used the nominal concentration

101 below. Tubes were placed in a randomized fashion inside a dark temperature-controlled

102 incubator at $20^{\circ} \mathrm{C}$ for 6 weeks, and each tube was watered weekly to maintain soil moisture.

103 This experiment ran with 10 replications of blank control (without any PFAS added, but

104 handled exactly the same way) and 8 replications of each treatment, for a total of 106

105 microcosms.

106 Proxies for soil health and function

107 We measured well-established proxies for soil health and functions, including soil respiration,

108 litter decomposition, enzyme activities, soil aggregates, and soil bacterial abundance. Soil

109 respiration was measured with an infrared gas analyzer (LI-6400XT, LI-COR Inc., Bad

110 Homburg, Germany), and litter decomposition was determined by the mass loss of tea bags

$111{ }^{18}$. Soil enzymes activities were measured, including four enzymes concerning $C$ ( $\beta$ -

112 glucosidase and $\beta$-D-1,4-cellobiosidase), $N(\beta-1,4-N$-acetyl-glucosaminidase), and $P$

113 (phosphatase) cycling, and fluorescein diacetate hydrolase (FDA) representing general soil

114 microbial activity. Water-stable aggregates, as the basic unit of soil structure, were qualified

115 using a wet-sieving apparatus (Eijkelkamp, Giesbeek, Netherlands) with an established 
116 method $^{22,23}$. Soil DNA was extracted with DNeasy PowerSoil Pro Kit (QIAGEN GmbH,

117 Germany) following the technical protocol, and we amplified using the universal primers

118 515F (5'-GTGCCAGCMGCCGCGGTAA-3') and 806R (5'-GGACTACHVGGGTWTCTAAT-3')

119 with quantitative polymerase chain reactions (qPCR) in a CFX 96 Real-Time System (Bio-

120 Rad Lab., Hercules, USA). For more information on measurement procedures, qPCR

121 conditions and quality control, see SI Text S2.

\section{Statistical analysis}

123 All statistical analyses and data visualization were performed in $\mathrm{R}^{24}$. The effects of PFAS

124 treatment (four concentrations per PFAS type) on soil functions were tested with a two-step

125 method. Firstly, we calculated the $95 \%$ confidence interval $(\mathrm{Cl})$ of unpaired mean differences

126 (treatment minus control) using the R package "dabestr" ${ }^{25}$. This approach focuses on the

127 effect size and its precision, and can avoid the pitfalls of significance testing. Secondly, one-

128 way analysis of variance (ANOVA) followed by Dunnett's test in the R package "multcomp"

129 was implemented to compare each treatment with the control ${ }^{26}$. Model residuals were

130 checked for heteroscedasticity and normal distribution. Spearman correlations among actual

131 concentrations of PFAS and soil structure and function were performed with the package

132 "corrplot" 27 . Adjusted p values by a single-step method are reported in the SI Table S3. All

133 data used for analyses and plotting are available online ${ }^{28}$, and plots were generated with the

134 package "ggplot2" 29.

\section{Results and Discussion}

\section{PFAS increased litter decomposition associated with bacterial abundance}

137 Positive effects of three PFASs on litter decomposition were observed (Figure 1A), and

138 PFBS, particularly, at all tested concentrations significantly increased the decomposition rate

$139(p<0.05$, Table S3). Regardless of tested type, increasing concentrations of PFAS

140 significantly enhanced litter decomposition ( $r=0.15, p=0.0221$, Figure S1 and S2B). In

141 terms of PFAS type, PFOA and PFBS resulted in significantly positive effects on litter 
142 decomposition ( $p<0.01$, Table S3), and PFBS exerted the most remarkable positive effect

143 (Figure S1C).

144 The treatment of PFASs significantly increased soil bacterial abundance $(\mathrm{F}=1.868, p=$

145 0.0485), although the multiple comparison tests did not reveal a significant difference (Table

146 S3). PFBS seems to have the most obvious effect among three PFAS, showing a positive

147 effect with concentrations higher than $0.5 \mathrm{ng} \mathrm{g}^{-1}$ (Figure 1B). Independently of concentration,

148 PFAS treatments had a positive effect on soil bacterial abundance in terms of copy number

149 per gram of dry soil (Figure S3C).

150 Microorganisms, including bacteria and fungi, play an essential role in the biological

151 decomposition of organic matter in soil, a process in which bacteria generally predominate in

152 neutral or alkaline soils, while fungi are more important in acidic soil ${ }^{30,31}$. Correspondingly,

153 we found that in our $\mathrm{pH}$-neutral soil, with various PFAS treatments, the number of bacteria

154 increased compared to the control (Figure 1B), and there was a trend for a correlation

155 between bacteria abundance and litter decomposition rate $(\mathrm{F}=2.705, p=0.103)$. The

156 increased bacterial populations probably contributed to the increased decomposition rate of

157 organic matter.

158 Litter decomposition and the ensuing nutrient release, governing carbon and nutrient cycling,

159 is a key process in terrestrial ecosystems. Our results showed that PFAS had a positive

160 effect on litter decomposition, and particularly the PFBS treatment, even at $1 \mathrm{ng} \mathrm{g}^{-1}$ in soil,

161 resulting in a significant enhancement of litter decomposition, likely associated with

162 increased soil bacterial abundance. 


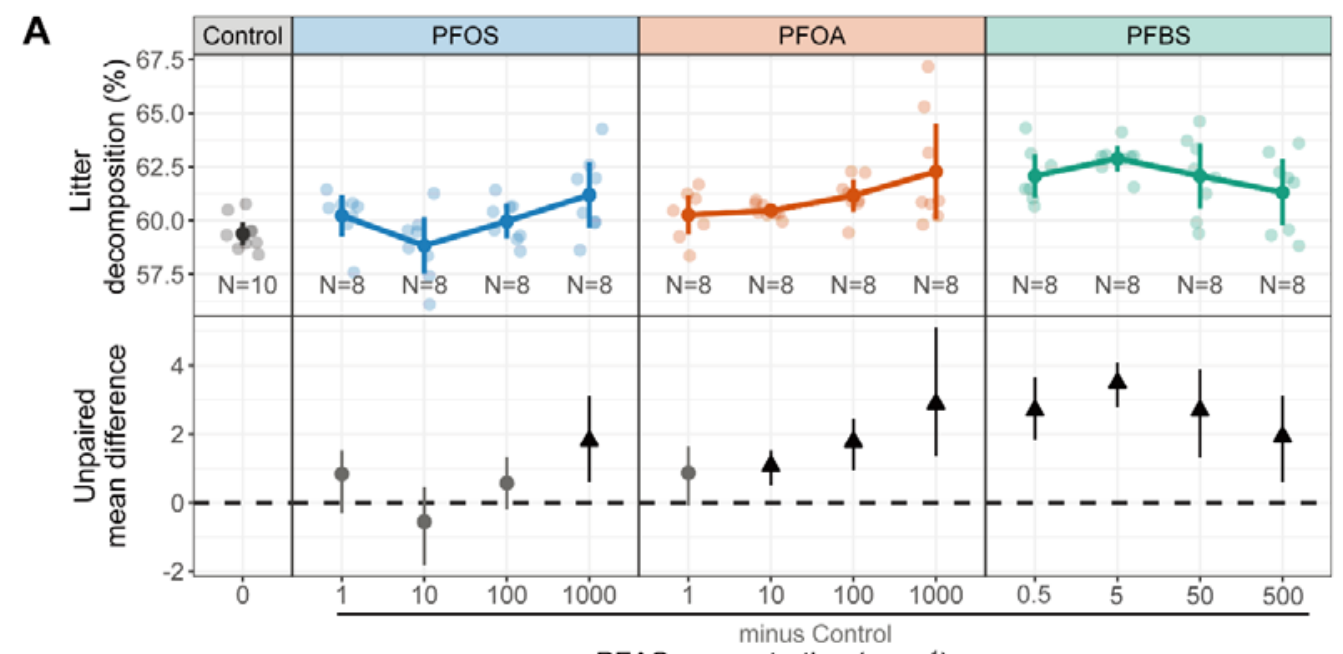

PFAS concentration ( $\mathrm{ng} \mathrm{g}^{-1}$ )

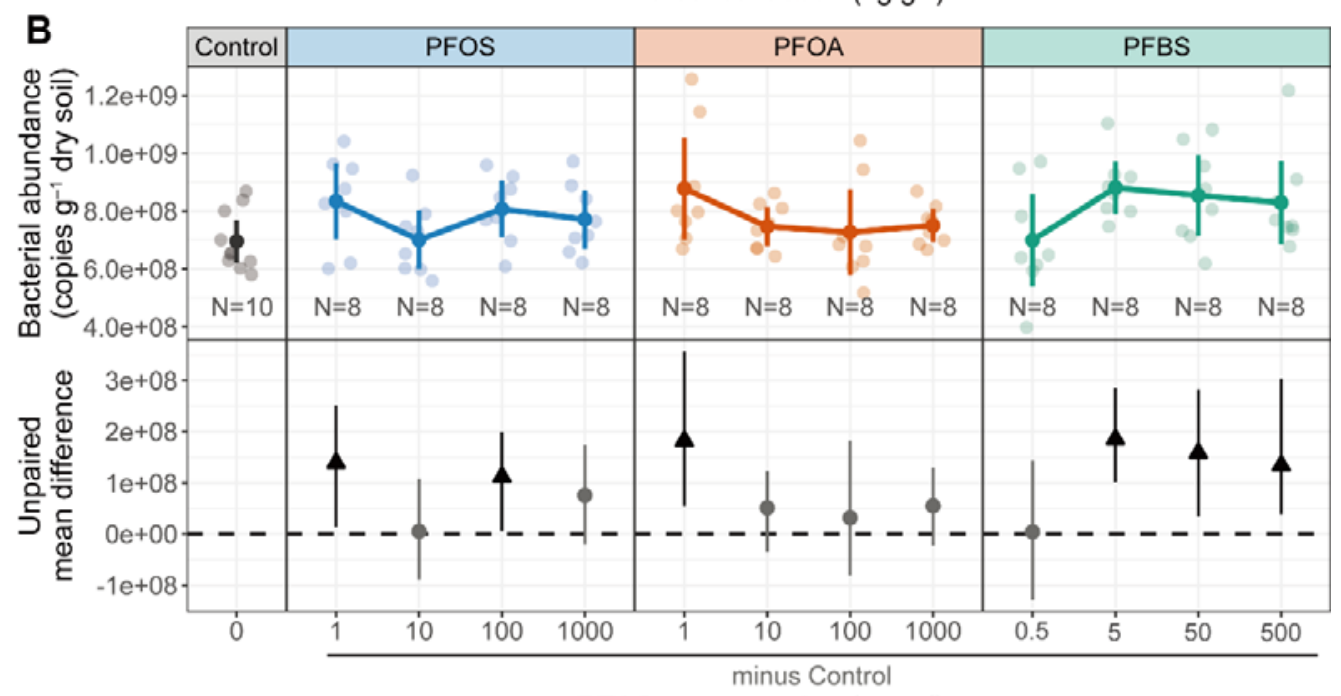

165 Figure 1. Effects of per- and polyfluoroalkyl substances (PFAS) on litter decomposition (A) and bacterial abundance $(B)$ in soil. In the first row of each panel, raw data are presented as

167 both scatter points and the corresponding mean and $95 \%$ confidence intervals (Cls) $(\mathrm{N}=8$

168 for each treatment, and $\mathrm{N}=10$ for blank control). In the second row, estimation plots present

169 the unpaired mean difference between each treatment and the shared control. Circles in

170 grey and triangles (arrow head up) in black represent neutral and positive effects,

171 respectively. PFOS, perfluorooctanesulfonic acid; PFOA, perfluorooctanoic acid; PFBS,

172 perfluorobutanesulfonic acid. Summary of effects of PFAS concentration or type refers to SI

173 Figure S2 and S3, respectively, and outcomes of ANOVA followed by Dunnett's test is 174 presented in SI Table S3. 


\section{Soil respiration is inhibited by PFAS}

176 We observed that the tested PFAS produced significantly negative effects on soil respiration

177 in week 3 (Figure 2A and S4), while more variable effects were present in week 6 (Figure

178 S5). In week 3, PFOA and PFBS at all tested concentrations exerted negative effects on soil

179 respiration, while effects of PFOS were dependent on its concentration $(F=5.461, p<$

180 0.001). Irrespective of concentration, our tested PFAS had negative effects on soil

181 respiration in week 3 (Figure S4C). Compared with soil respiration in week 3, less

182 pronounced effects were observed in week 6 . Similar to effects in week 3 , PFBS had the

183 most apparent effect on respiration in week 6 (Figure S5C). However, this negative effect

184 was significant only at its highest concentration (500 $\mathrm{ng} \mathrm{g}^{-1}, p=0.019$, Table S3).

185 Waning effects of organic compounds on soil respiration during the incubation period have

186 been previously reported ${ }^{32,33}$. For example, the fungicides tebuconazole and carbendazim

187 significantly suppressed soil respiration during the first 30 days, while this effect was no

188 longer present on the $90^{\text {th }}$ day ${ }^{34}$. Microorganisms would react to the exogenous PFAS

189 during the first stage, expressed as an inhibitory effect, and gradually recover from this

190 inhibition during the incubation. Another possible explanation is that as litter decomposition

191 progressed, easily available carbon (e.g., sugars) was progressively utilized by microbes

192 and subsequently transferred to $\mathrm{CO}_{2}$, which modulated the suppressed production of $\mathrm{CO}_{2}$. 

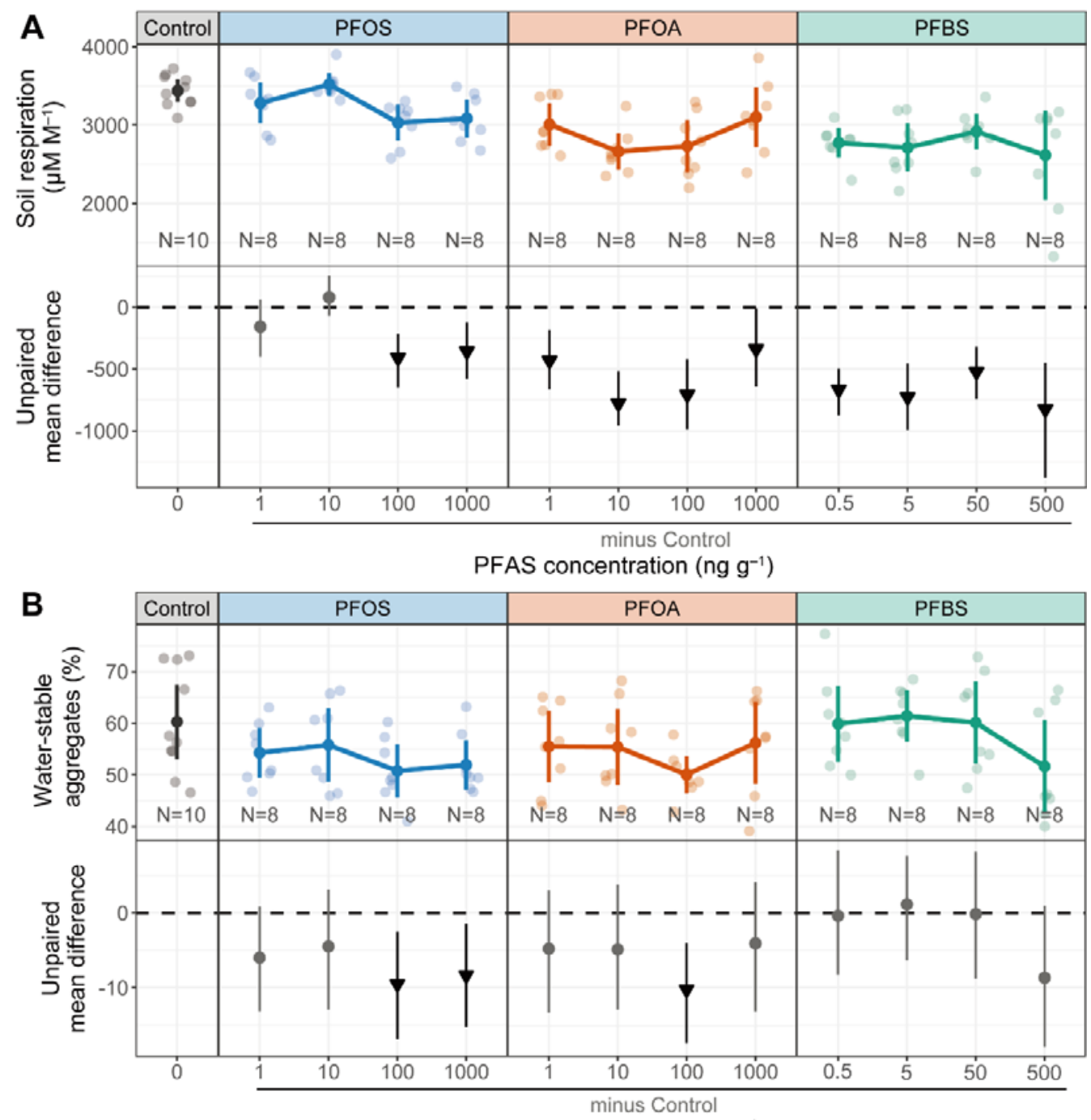

195 Figure 2. Effects of per- and polyfluoroalkyl substances (PFAS) on soil respiration at the $3^{\text {rd }}$

196 week (A) and water-stable aggregates (B). In the first row of each panel, raw data are

197 presented as both scatter points and the corresponding mean and 95\% confidence intervals

198 (Cls) $(\mathrm{N}=8$ for each treatment, and $\mathrm{N}=10$ for blank control). In the second row, estimation

199 plots present the unpaired mean difference between each treatment and the shared control.

200 Circles in grey and triangles (arrow head down) in black represent neutral and negative

201 effects, respectively. PFOS, perfluorooctanesulfonic acid; PFOA, perfluorooctanoic acid;

202 PFBS, perfluorobutanesulfonic acid. Soil respiration at the $6^{\text {th }}$ week refers to SI Figure S5.

203 Summary of effects of PFAS concentration or type refers to SI Figure S4 and S6, and

204 outcomes of ANOVA followed by Dunnett's test is presented in SI Table S3. 


\section{Limited effects on soil enzyme and microbial activities}

206 Four enzymes were not significantly affected by individual PFAS treatments (Figures S7-

207 S10), nor the general microbial activities (Figure S11, Table S3), but regardless of

208 concentration, PFBS significantly increased $\beta$-glucosidase activity ( $p=0.029$, Figure S9C).

209 Measuring enzyme activities provides evidence on how soil biochemical processes might be

210 affected. $\beta$-glucosidase is responsible for catalyzing the hydrolysis of cellobiose (a product of

211 cellulose breakdown) to glucose ${ }^{35}$. We observed a positive trend on $\beta$-glucosidase by

212 PFASs, particularly PFBS, which probably contributed to the litter decomposition by PFASs.

213 The only significant effect on $\beta$-glucosidase corresponded with the most marked effect on

214 litter decomposition by PFBS. Additionally, there was a significant correlation between

215 decomposition rate and $\beta$-glucosidase activity $(r=0.24, p=0.004)$ (Figure $S 1)$.

216 Previous studies reported that soil dehydrogenase (proxy for total microbial activity), urease

217 and sucrase activities were only insignificantly impacted by PFOA and PFOS with

218 concentrations lower than $10 \mu \mathrm{g} \mathrm{g}^{-1} 12,36$. Cai et al. ${ }^{13}$ also reported that microbial activity was

219 barely affected by PFAS at $100 \mathrm{\mu g} \mathrm{g}^{-1}$ in selected soils. Changes in enzyme activities are

220 highly dynamic processes ${ }^{12,37}$, and thus insignificant effects observed at harvest do not

221 necessarily indicate that there were no remarkable changes during the incubation. In

222 addition, there was no significant relationship between microbial activities (FDA) with other

223 parameters (Figure S1). Overall, general microbial activities were affected only to a very

224 limited degree.

\section{Different effect sizes caused by three PFAS}

226 The three PFAS examined here appeared to exert similar impact on soil microbes and

227 functions, but with different effect sizes, which is likely related to their bioavailability and

228 bioaccumulation ${ }^{8}$. Of the three PFASs, PFBS even at lower concentrations seemed to have

229 the most remarkable impact on soil respiration, litter decomposition and soil bacterial

230 abundance, while PFOS had a larger effect size on water-stable aggregates. 
231 Sorption affinities of PFASs followed the order PFOS $>$ PFOA > PFBS on soils with various

232 soil textures and organic carbon contents, showing the same order of their hydrophobicity ${ }^{20}$.

233 With a low sorption affinity to soil particles, PFBS likely had an increased likelihood to

234 interact with soil microbes, subsequently causing an impact. However, it is not a simple

235 effect of hydrophobicity, because, for example, the higher hydrophobicity might result in

236 higher bioaccumulation and hence exert higher toxicity in soil microorganisms ${ }^{12,13}$. This

237 might explain the more apparent effect by PFOS on some processes via soil microbes.

\section{Environmental implications and future perspectives}

239 The present results highlight the potential of PFAS to induce changes in soil properties and

240 functions. Our study comprehensively analyzed PFAS impacts on soil functions, and thus we

241 hope that our result inspires further studies that consider the impact of PFAS on soil

242 ecosystem functions.

243 Within environmentally relevant concentrations, three PFAS, especially the short-chain

244 PFBS, had a positive effect on litter decomposition in neutral soil. This effect indicates that

245 the PFAS present in soils now might already affect ecosystem processes. The elevated

246 decomposition might increase the release of carbon as $\mathrm{CO}_{2}, \mathrm{CH}_{4}$ and dissolved organic

247 carbon, affecting carbon sinks in soil ${ }^{38}$. The precise mechanisms underpinning litter

248 decomposition effects caused by PFAS need to be explored in further studies, with changes

249 in microbial community composition likely playing a main role. In addition, effects are likely

250 influenced by soil and PFAS properties.

251 Soil aggregation is an essential feature of soil structure, principally driven by soil biota and

252 their interactions ${ }^{39}$. Our finding that certain PFAS negatively affected water-stable

253 aggregates could indicate far-reaching consequences for soil health, given the many

254 influences of soil structure on virtually all soil processes. Thus, future studies might explore

255 these effects on the soil aggregation process in greater depth, including the formation, size

256 distribution of soil aggregates and their intrinsic connections with soil biota. 
bioRxiv preprint doi: https://doi.org/10.1101/2021.10.26.465889; this version posted November 10,2021 . The copyright holder for this preprint

(which was not certified by peer review) is the author/funder, who has granted bioRxiv a license to display the preprint in perpetuity. It is made available under aCC-BY-NC-ND 4.0 International license.

257 We introduce the possibility of PFAS as persistent chemicals being a potential environmental

258 change factor. The effects of various PFAS on soil functions should now be addressed in the

259 context of global patterns of contamination.

260

261 Acknowledgements

262 BX thanks the China Scholarship Council and Deutscher Akademischer Austauschdienst

263 (CSC-DAAD) for a postdoctoral scholarship. MCR acknowledges support from an ERC

264 Advanced Grant (694368). We thank Daniel Lammel, Yun Liang, Tingting Zhao, and Lili

265 Rong for their help with experimental measurements. We thank Rosolino Ingraffia for

266 providing soil samples. 


\section{References}

269 (1) Zhu, Y. G.; Penuelas, J. Changes in the Environmental Microbiome in the Anthropocene. Glob. Chang. Biol. 2020, 26 (6), 3175-3177. https://doi.org/10.1111/gcb.15086.

272 (2) Morgado, R. G.; Loureiro, S.; González-Alcaraz, M. N. Changes in Soil Ecosystem Structure and Functions Due to Soil Contamination. Soil Pollut. From Monit. to Remediat. 2018, 59-87. https://doi.org/10.1016/B978-0-12-849873-6.00003-0.

Rillig, M. C.; Ryo, M.; Lehmann, A. Classifying Human Influences on Terrestrial Ecosystems. Glob. Chang. Biol. 2021, gcb.15577. https://doi.org/10.1111/gcb.15577.

(4) Lim, X. Tainted Water: The Scientists Tracing Thousands of Fluorinated Chemicals in Our Environment. Nature 2019, 566 (7742), 26-29. https://doi.org/10.1038/d41586019-00441-1.

(5) Buck, R. C.; Franklin, J.; Berger, U.; Conder, J. M.; Cousins, I. T.; Voogt, P. De; Polyfluoroalkyl Substances in the Environment: Terminology, Classification, and Origins. Integr. Environ. Assess. Manag. 2011, 7 (4), 513-541. https://doi.org/10.1002/ieam.258.

(6) Beans, C. News Feature: How "Forever Chemicals" Might Impair the Immune System.

Proc. Natl. Acad. Sci. 2021, 118 (15). https://doi.org/10.1073/PNAS.2105018118.

287 (7) UNEP. Stockholm Convention on Persistent Organic Pollutants (POPS) - Texts and Annexes. United Nations Environ. Program. 2019.

289 (8) Cai, Y.; Wang, Q.; Zhou, B.; Yuan, R.; Wang, F.; Chen, Z.; Chen, H. A Review of 2021, 793. https://doi.org/10.1016/j.scitotenv.2021.148565. 
292 (9) Ma, D.; Zhong, H.; Lv, J.; Wang, Y.; Jiang, G. Levels, Distributions, and Sources of 293 Legacy and Novel per- and Perfluoroalkyl Substances (PFAS) in the Topsoil of Tianjin, 294 China. J. Environ. Sci. 2022, 112, 71-81. https://doi.org/10.1016/J.JES.2021.04.029.

295 (10) Brusseau, M. L.; Anderson, R. H.; Guo, B. PFAS Concentrations in Soils: Background 296 Levels versus Contaminated Sites. Sci. Total Environ. 2020, 740, 140017. https://doi.org/10.1016/j.scitotenv.2020.140017.

(11) Jin, H.; Zhang, Y.; Zhu, L.; Martin, J. W. Isomer Profiles of Perfluoroalkyl Substances in Water and Soil Surrounding a Chinese Fluorochemical Manufacturing Park. Environ. Sci. Technol. 2015, 49 (8), 4946-4954. https://doi.org/10.1021/acs.est.5b00212.

(12) Qiao, W.; Xie, Z.; Zhang, Y.; Liu, X.; Xie, S.; Huang, J.; Yu, L. Perfluoroalkyl Substances (PFASs) Influence the Structure and Function of Soil Bacterial Community: Greenhouse Experiment. Sci. Total Environ. 2018, 642, 1118-1126. https://doi.org/10.1016/j.scitotenv.2018.06.113.

(13) Cai, Y.; Chen, H.; Yuan, R.; Wang, F.; Chen, Z.; Zhou, B. Toxicity of Perfluorinated Compounds to Soil Microbial Activity: Effect of Carbon Chain Length, Functional Group and Soil Properties. Sci. Total Environ. 2019, 690, 1162-1169.

(14) Xu, R.; Tao, W.; Lin, H.; Huang, D.; Su, P.; Gao, P.; Sun, X.; Yang, Z.; Sun, W. Effects of Perfluorooctanoic Acid (PFOA) and Perfluorooctane Sulfonic Acid (PFOS)

(15) Cai, Y.; Chen, H.; Yuan, R.; Wang, F.; Chen, Z.; Zhou, B. Metagenomic Analysis of Soil Microbial Community under PFOA and PFOS Stress. Environ. Res. 2020, 188 (May), 109838. https://doi.org/10.1016/j.envres.2020.109838. 
316 (16) Chen, H.; Wang, Q.; Cai, Y.; Yuan, R.; Wang, F.; Zhou, B.; Chen, Z. Effect of per FI

317 Uorooctanoic Acid on Microbial Activity in Wheat Soil under Different Fertilization

318 Conditions. Environ. Pollut. 2020, 264, 114784.

319 https://doi.org/10.1016/j.envpol.2020.114784.

320 (17) Rillig, M. C.; Mardatin, N. F.; Leifheit, E. F.; Antunes, P. M. Mycelium of Arbuscular Mycorrhizal Fungi Increases Soil Water Repellency and Is Sufficient to Maintain Water-Stable Soil Aggregates. Soil Biol. Biochem. 2010, 42 (7), 1189-1191. https://doi.org/10.1016/j.soilbio.2010.03.027.

(18) Lehmann, A.; Leifheit, E. F.; Feng, L.; Bergmann, J.; Wulf, A.; Rillig, M. C. Microplastic Fiber and Drought Effects on Plants and Soil Are Only Slightly Modified by Arbuscular Mycorrhizal Fungi. Soil Ecol. Lett. 2020. https://doi.org/10.1007/s42832-020-0060-4.

(19) Ahmed, M. B.; Johir, M. A. H.; McLaughlan, R.; Nguyen, L. N.; Xu, B.; Nghiem, L. D. Per- and Polyfluoroalkyl Substances in Soil and Sediments: Occurrence, Fate, Remediation and Future Outlook. Sci. Total Environ. 2020, 748, 141251. https://doi.org/10.1016/j.scitotenv.2020.141251.

(20) Milinovic, J.; Lacorte, S.; Vidal, M.; Rigol, A. Sorption Behaviour of Perfluoroalkyl

(21) Rillig, M. C.; Ryo, M.; Lehmann, A.; Aguilar-Trigueros, C. A.; Buchert, S.; Wulf, A.; Iwasaki, A.; Roy, J.; Yang, G. The Role of Multiple Global Change Factors in Driving Soil Functions and Microbial Biodiversity. Science (80-. ). 2019, 366 (6467), 886-890. https://doi.org/10.1126/science.aay2832. 
341 (23) Liang, Y.; Lehmann, A.; Ballhausen, M.-B.; Muller, L.; Rillig, M. C. Increasing

342 Temperature and Microplastic Fibers Jointly Influence Soil Aggregation by Saprobic

343 Fungi. Front. Microbiol. 2019, 10 (September), 1-10.

$344 \quad$ https://doi.org/10.3389/fmicb.2019.02018.

345 (24) R Core Team. R: A Language and Environment for Statistical Computing. Vienna 3462020.

347 (25) Ho, J.; Tumkaya, T.; Aryal, S.; Choi, H.; Claridge-Chang, A. Moving beyond P Values:

348 Data Analysis with Estimation Graphics. Nat. Methods 2019, 16 (7), 565-566.

$349 \quad$ https://doi.org/10.1038/s41592-019-0470-3.

350 (26) Hothorn, T.; Bretz, F.; Westfall, P. Simultaneous Inference in General Parametric Models. Biometrical Journal. John Wiley \& Sons, Ltd June 1, 2008, pp 346-363. https://doi.org/10.1002/bimj.200810425.

(27) Wei, T.; Simko, V. R Package "Corrplot”: Visualization of a Correlation Matrix. 2017.

(28) Xu, B.; Yang, G.; Lehmann, A.; Hasenstab-Riedel, S.; Rillig, M. C. PFAS Effects on Soil Structure and Function. 2021. https://doi.org/10.6084/m9.figshare.16967401.v1.

(29) Wickham, H. Ggplot2: Elegant Graphics for Data Analysis; Springer-Verlag: New York, 2016.

358

(30) Valentín, L.; Nousiainen, A.; Mikkonen, A. Introduction to Organic Contaminants in Soil: Concepts and Risks; 2013; pp 1-29. https://doi.org/10.1007/698_2012_208.

360 (31) Rousk, J.; Brookes, P. C.; Bååth, E. Investigating the Mechanisms for the Opposing PH Relationships of Fungal and Bacterial Growth in Soil. Soil Biol. Biochem. 2010, 42 (6), 926-934. https://doi.org/10.1016/j.soilbio.2010.02.009. into Dose- and Time-Dependent Response of Five Typical PPCPs on Soil Microbial 
365 Respiration. Bull. Environ. Contam. Toxicol. 2019, 103 (1), 193-198.

366 https://doi.org/10.1007/s00128-019-02655-5.

367 (33) Xiong, D.; Li, Y.; Xiong, Y.; Li, X.; Xiao, Y.; Qin, Z.; Xiao, Y. Influence of Boscalid on 368 the Activities of Soil Enzymes and Soil Respiration. Eur. J. Soil Biol. 2014, 61, 1-5. 369 https://doi.org/10.1016/j.ejsobi.2013.12.006.

370 (34) Wang, C.; Wang, F.; Zhang, Q.; Liang, W. Individual and Combined Effects of Tebuconazole and Carbendazim on Soil Microbial Activity. Eur. J. Soil Biol. 2016, 72, 6-13. https://doi.org/10.1016/j.ejsobi.2015.12.005.

(35) German, D. P.; Weintraub, M. N.; Grandy, A. S.; Lauber, C. L.; Rinkes, Z. L.; Allison,

(36) He, W.; Megharaj, M.; Naidu, R. Toxicity of Perfluorooctanoic Acid towards Earthworm and Enzymatic Activities in Soil. Environ. Monit. Assess. 2016, 188 (7). https://doi.org/10.1007/s10661-016-5416-y.

(37) Zhao, T.; Lozano, Y. M.; Rillig, M. C. Microplastics Increase Soil PH and Decrease Microbial Activities as a Function of Microplastic Shape, Polymer Type, and Exposure Time. Front. Environ. Sci. 2021, 9, 1. https://doi.org/10.3389/fenvs.2021.675803. Ecol. Evol. 2017, 1 (12), 1828-1835. https://doi.org/10.1038/s41559-017-0344-y. 